\section{Atividade física e saúde mental: uma experiência na formação inicial em Educação Física}

\section{Physical activity and mental health: an experience in an undergraduate Physical Education program}

\author{
Juliana Rafaela Andrade da Silva' \\ Maria Laura Siqueira de Souza Andrade ${ }^{1}$ \\ Anísio Luiz da Silva Brito' \\ Carla Menêses Hardman \\ Elusa Santina Antunes de Oliveira \\ Mauro Virgílio Gomes de Barros
}

\section{RESUMO}

O objetivo deste relato foi descrever uma experiência curricular inovadora realizada no curso de bacharelado em Educação Física da Universidade de Pernambuco. A disciplina "Atividade Física e Saúde Mental” foi implantada no referido curso para suprir a lacuna existente entre a formação inicial e a intervenção profissional no campo da saúde mental.A proposta desta disciplina foi discutir o papel da atividade física na prevenção e tratamento dos transtornos mentais, bem com a intervenção profissional na rede de saúde mental. As ações realizadas na disciplina foram pautadas por uma perspectiva crítica no sentido de provocar nos estudantes um olhar diferenciado acerca desse campo de atuação. A disciplina possibilitou ainda a observação e a vivência da intervenção da Educação Física nos Centros de Atenção Psicossocial, aspecto diferencial na organização do componente curricular.

\section{PALAVRAS-CHAVE}

Currículo; Atividade física; Saúde mental.

\begin{abstract}
The aim of this report was to describe an innovative curricular experience that was performed in the bachelor in Physical Education offered by the University of Pernambuco (Brazil). A course entitled "Physical Activity and Mental Health" was offered to the bachelor students with the idea of fulfill the existing gap between the professional preparation and the intervention in the mental health care area. The course was organized to discuss the role of physical activity in the prevention and control of mental illness as well as the professional intervention of physical educators in this field. Activities performed during the lessons were guided by a critical pedagogy approach which means the students were challenged to think about this field of intervention in different ways. In addition, the course offered the opportunity for extramural observational and experiential activities in specialized centers of primary mental bealth care, a differential characteristic of this proposed curricular component.
\end{abstract}

\section{KEYWORDS}

Curriculum; Motor Activity; Mental Health.
Rev Bras Ativ Fis Saúde p. 133-140 $\mathrm{DOI}$

http://dx.doi.org/10.12820/rbafs.v.19n1p133

1 Universidade de Pernambuco. Grupo de Pesquisa em Estilos de Vida e Saúde. Programa Associado de Pós-graduação em Educação Física UPE/UFPB. Programa de Pós-graduação em Hebiatria. Recife, PE, Brasil. 


\section{INTRODUÇÃOO}

Em 2006, a partir de uma iniciativa conjunta dos Ministérios da Saúde e da Educação, especialistas foram desafiados a examinar dados sobre a trajetória dos cursos de graduação das diversas áreas da saúde. Ao final de inúmeras reuniões, a comissão constituída para analisar os dados relativos à área da Educação Física (EF) apresentou um relatório no qual ficou destacada a expansão desordenada da oferta de cursos e vagas nas diferentes regiões brasileiras, observada a partir de $1999^{1}$. A comissão revelou ainda a crença de que as diretrizes curriculares para os cursos de $\mathrm{EF}$, que passavam a vigorar naquele período, teriam impacto na adequação da formação inicial às demandas sociais, particularmente em relação ao setor saúde. Esta expectativa estava fundamentada, principalmente, na observação do intenso processo de reformulação curricular que ocorria nas instituições de ensino superior brasileiras à época.

Havia também a expectativa de que as reformas curriculares concorreriam para promoção de melhorias substanciais na formação inicial, permitindo uma maior integração do profissional de EF às ações do Sistema Único de Saúde. Não há dados para julgar se esta expectativa se concretizou, mas parece inequívoco que ainda existe um importante hiato entre as atividades de formação e a realidade da intervenção profissional em saúde. Apesar de todas as oportunidades criadas para aproximar a formação inicial em EF das demandas vivenciadas na intervenção profissional na Atenção Básica à Saúde (ABS), a exemplo do Programa de Educação pelo Trabalho para a Saúde, a atenção à saúde mental (ASM) continua sendo um tema negligenciado nessa área de formação inicial.

Foi diante desta lacuna que em 2012, o Grupo de Pesquisa em Estilos de Vida e Saúde da Universidade de Pernambuco começou a discutir as possibilidades para enfrentamento deste desafio. Depoimentos de residentes em saúde mental e em saúde da família forneceram os elementos iniciais para a compreensão das principais barreiras enfrentadas pelos mesmos ao ingressar na realidade da intervenção profissional. Com base nestes dados preliminares, partiu-se para a realização de uma experiência piloto cujo objetivo seria oferecer oportunidades para desenvolvimento de competências conceituais e atitudinais básicas necessárias à aproximação dos estudantes de graduação em $\mathrm{EF}$ da realidade da intervenção profissional na ASM. O objetivo deste relato é apresentar uma análise desta experiência curricular inovadora, abordando suas características, dificuldades e avanços observados.

\section{ATUAC̣ÃO DA EF NA SAÚDE MENTAL: VISÃO GERAL}

No Brasil, o atual modelo de ASM, pautado na integralidade e na atuação multiprofissional, abriu caminhos para a inclusão de práticas sanitárias dife-

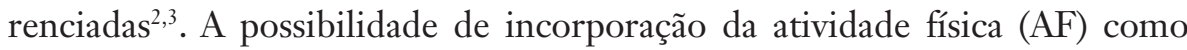
componente do chamado "projeto terapêutico singular" ampliou o espectro de oportunidades para inserção do profissional de EF nos serviços de ASM. Mas, apesar disto, tal intervenção continua acontecendo de modo bastante incipiente e desordenado.

É possível dizer que no contexto da intervenção, os profissionais de EF não constituem, via de regra, categoria integrante das equipes responsáveis 
pelo desenvolvimento das práticas terapêuticas, limitando-se a atuar de modo complementar e eventual ${ }^{4,5}$. Isto decorre, ao menos em parte, de dois fatores: o reduzido corpo de conhecimento decorrente da escassez de estudos sobre a temática; e, a ausência da tematização da saúde mental na formação inicial. O pouco que se conhece sobre o assunto é derivado de investigações nas quais se procurou analisar os efeitos da AF sobre indicadores de saúde mental ou verificar associações entre estes fatores. Além disso, questões como a formação e a atuação do profissional de EF na ASM permanecem ainda pouco investigadas ${ }^{6}$.

Nos currículos dos cursos de EF, a inclusão de disciplinas que problematizem a atuação profissional na saúde mental pode ser o primeiro passo para reduzir o hiato entre a formação e a intervenção profissional. Além disso, a vivência de estágios nos serviços de ASM e a participação em projetos de pesquisa e de extensão podem atenuar as barreiras enfrentadas pelos profissionais ao ingressarem no mercado de trabalho. Na formação continuada, a ampliação do número de vagas nos programas de residência em saúde mental poderá garantir uma qualificação ainda mais especializada, mas há de se reconhecer que é na formação inicial que este processo deve ter início.

Em termos práticos, as $\mathrm{AF}$ assumem, quase sempre, um caráter complementar no projeto terapêutico. Não raras vezes tais atividades são utilizadas apenas como uma estratégia para tornar o ambiente dos serviços de saúde mental mais agradável tanto para os pacientes quanto para os demais profissionais ${ }^{6}$. Em parte, a subvalorização ou subutilização das AF como parte dos recursos terapêuticos na ASM pode ser decorrente do desconhecimento quanto aos benefícios que tais práticas podem derivar ${ }^{7-9}$.

\section{DESCREVENDO UMA EXPERIÊNCIA CURRICULAR INOVADORA}

O projeto curricular do curso de bacharelado em EF da Escola Superior de Educação Física da Universidade de Pernambuco foi construído considerando princípios norteadores, bases para a dinâmica curricular e contextos de intervenção (esporte, lazer e saúde) ${ }^{10}$. Respeitados estes elementos, definiuse um projeto pedagógico de curso (PPC) que compreende uma formação ampliada e outra específica. No contexto da saúde, a formação específica será orientada para o desenvolvimento de competências relativas à intervenção na prevenção, promoção, proteção e reabilitação da saúde individual e coletiva.

O PPC prevê a realização de uma série de ações curriculares orientadas para a preparação de profissionais capazes de intervir profissionalmente no campo da saúde. Aspecto que por si só representa, sem dúvidas, importante avanço no tocante à formação inicial em EF. Todavia, poucos componentes curriculares permitem efetivamente uma aproximação teórico-metodológica em relação à realidade da intervenção na $\mathrm{ABS}$ e nenhum focalizava a questão da saúde mental.

A disciplina de "Atividade Física e Saúde Mental" foi implantada no referido curso para suprir esta lacuna (Figura 1). A proposta inicial era de oferecer uma disciplina optativa na qual seriam discutidas, por uma abordagem predominantemente teórica, questões relativas ao papel que as AF poderiam assumir na prevenção e tratamento de transtornos mentais com maior incidência na população. Mas, restava ainda a aproximação destes conhecimentos em relação ao contexto de intervenção profissional. 


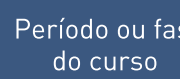

do curso
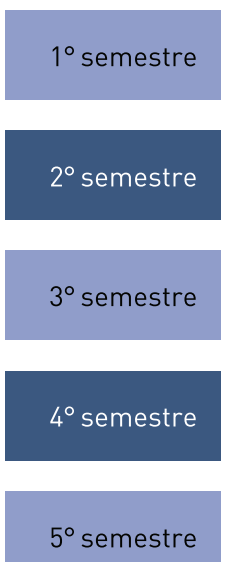

\section{$6^{\circ}$ semestre}

$7^{\circ}$ semestre
Componente curricular obrigatório

Fundamentos acadêmicos e profissionais da Educação Física*

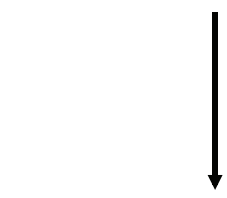

Módulo interprofissional* “Atenção à saúde na perspectiva do cuidado, integralidade da atenção e trabalho em equipe"

$8^{\circ}$ semestre

Intervenções da Educação Física na saúde*

$\downarrow$

\section{Estágio supervisionado em saúde (ou Estágio I)*}

FIGURA 1 - Representação esquemática da oferta de disciplinas e estágios relacionados ao núcleo temático "saúde" no curso de bacharelado em Educação Física da ESEF/UPE.

* Disciplina oferecida a todos os estudantes de saúde como estratégia para iniciar entendimento sobre o processo de trabalho em equipe, na perspectiva da integralidade e cuidado na atenção à saúde em rede.

** As disciplinas optativas oferecidas aos estudantes abrangem as seguintes opções: (1) Avaliação, prescrição e supervisão de programas de exercícios físicos; (2) Teoria e prática das atividades em academias; (3) Promoção da saúde e de estilo de vida saudável; (4) Programas clínicos e ambulatoriais de exercício físico; e (5) Tópicos avançados em saúde.

*** 0 estágio supervisionado IV pode ser realizado em qualquer um dos núcleos temáticos lesporte, lazer ou saúde), a critério do estudante.

O passo seguinte na construção da proposta foi identificar profissionais de EF que atuavam na ASM, visando estabelecer parcerias que permitissem aos estudantes a realização de visitas e observações. Na Secretaria de Saúde da Cidade do Recife, os profissionais de EF contratados por concurso público para atuação no conhecido Programa Academia da Cidade (PAC) ${ }^{11}$ são encarregados de oferecer o suporte à rede de ASM. Por isso, a proposta de criação deste novo componente curricular passou a ser discutido tanto com a coordenação do PAC quanto com a coordenação da rede de ASM.

Deste debate entre academia e serviço surgiram os elementos básicos que permitiram a construção e, mais tarde, a efetiva realização desta experiência curricular inovadora, sob a forma de uma disciplina optativa e cujo modelo de plano de ensino está apresentado no Anexo 1. A carga horária destinada foi de 72 horas/aula, equivalente a aproximadamente $2 \%$ da carga horária mínima exigida para integralização do curso. Pode parecer pouco, mas certamente isto colocou definitivamente a "saúde mental" na agenda do debate sobre a formação inicial em EF na UPE.

A operação deste componente curricular prevê que 36 horas sejam destinadas ao debate teórico-metodológico relativo à intervenção no campo da saúde 
mental, a partir das AF, ou como está explicitado no próprio PPC, a partir das manifestações da cultura de movimento. As outras 36 horas seriam realizadas em um estágio nos Centros de Atenção Psicossocial, no qual os estudantes teriam a preceptoria dos profissionais que já atuam na rede. A intenção ao propor um estágio como parte integrante da disciplina foi garantir que esta vivência não ocorreria dissociada do necessário e salutar aprofundamento teórico dos problemas identificados no contexto vivencial, aproximando a pesquisa da ação.

Ao final de cada semestre, os estudantes, preceptores e docentes responsáveis pela disciplina participam de um seminário com o propósito de disseminar as experiências vivenciadas para toda comunidade acadêmica. Os relatos produzidos textualmente e apresentados oralmente neste seminário, aliada aos procedimentos de autoavaliação e de avaliação processual, constituem o principal mecanismo de avaliação pedagógica no qual estão envolvidos estudantes, preceptores e docentes. Do ponto de vista metodológico, as aulas e demais ações realizadas na disciplina foram pautadas por uma perspectiva crítica, na qual as atividades foram propostas com a intencionalidade de provocar nos estudantes um olhar diferenciado para as questões que envolvessem a saúde mental.

"Eu nunca me imaginei em um lugar como aquele, sempre tive medo e preconceitos com pessoas que possuem transtornos mais graves da mente. Mas com a convivência semanal junto aos usuários, passei a gostar de estar lá, de interagir com eles e com outros profissionais da saúde..."

\section{CONSIDERAÇÕES FINAIS}

Essa experiência curricular não pode ser entendida como um ponto de chegada, mas como um passo inicial para inclusão do debate acerca da saúde mental na formação inicial em EF. Há ainda muitos avanços a serem conquistados e o compartilhamento desta experiência pode estimular outras instituições a iniciarem ou aprofundarem o debate acerca da saúde mental nos cursos de graduação em EF. Assim, espera-se que docentes do ensino superior e profissionais de EF encontrem neste relato o incentivo à realização e disseminação de experiências congêneres.

\section{Agradecimentos}

Estudo apoiado com auxílio financeiro da Coordenação de Aperfeiçoamento de Pessoal de Nível Superior, do Conselho Nacional de Desenvolvimento Científico e Tecnológico e da Fundação de Amparo à Ciência e Tecnologia do Estado de Pernambuco.

\section{REFERÊNCIAS}

1. Hunger D, Nascimento JV, Barros MVG, Hallal PC. Educação Física. In: Haddad AE, Pierantoni CR, Ristoff D, Xavier IM, Giolo J, Silva LB. (Org.). A trajetória dos cursos de graduação na área da saúde: 1991-2004. Brasília: Instituto Nacional de Estudos e Pesquisas Educacionais Anísio Teixeira, 2006, p. 87-139.

2. Abib LT, Fraga AB, Wachs F, Alves CTP. Práticas corporais em cena na saúde mental: potencialidades de uma oficina de futebol em um centro de atenção psicossocial de Porto Alegre. Pensar prát. 2010; 13(2): 115.

3. Roble OJ, Moreira MIB; Scagliusi FB. A educação física na saúde mental: construindo uma formação na perspectiva interdisciplinar. Interface (Botucatu). 2012; 16(41): 567-78. 
4. Abib LT, Ferreira LAS. A cultura corporal no contexto da saúde mental. Rev Didática Sistêmica. 2010; Edição Especial: 11-23.

5. Ladvcat $\mathrm{MB}$, Teves $\mathrm{N}$. A representação do lugar social do profissional de educação física nos centros de atenção psicossocial infanto-juvenil. Arq Mov. 2011; 7(1): 34-51.

6. Wachs F. Educação física e o campo da saúde mental: uma reflexão introdutória. In: Fraga AB, Wachs F (eds.). Educação física e saúde coletiva: políticas de formação e perspectivas de intervenção. Porto Alegre: Editora da UFRGS; 2007. p.87-98.

7. Roeder MA. Benefícios da atividade física em pessoas com transtornos mentais. Rev bras ativ fís saúde. 1999; 4(2): 69-75.

8. Oliveira EN, Aguiar RC, Almeida MTO, Eloia SC, Lira TQ. Benefícios da atividade física para a saúde mental. Rev Saúde Coletiva [online]. 2011; 50(8):126-30. [acesso 2013 out 16]. Disponível em: http://www.redalyc.org/pdf/842/84217984006.pdf

9. Peluso MAM, Andrade LHSG. Physical activity and mental health: the association between exercise and mood. Clinics. 2005; 60(1): 61-70.

10. Universidade de Pernambuco. Escola Superior de Educação Física. Projeto Pedagógico do Curso de Bacharelado em Educação Física. Recife: ESEF/UPE; 2004.

11. Hallal PC, Tenório MCM, Tassitano RM, Reis RS, Carvalho YM, et al. Avaliação do programa de promoção da atividade física Academia da Cidade de Recife, Pernambuco, Brasil: percepções de usuários e não-usuários. Cad. Saúde Pública. 2010; 26(1): 70-78.

\section{ANEXO 1. MODELO DE PLANO DE ENSINO PARA DISCIPLINA DE ATIVIDADE FÍSICA E SAÚDE MENTAL}

\section{Justificativa}

As intervenções da Educação Física na área de saúde sofreram profunda modificação nos últimos anos, particularmente devido à ampliação das oportunidades de atuação no contexto do Sistema Único de Saúde. Uma das demandas sociais mais crescentes neste contexto é a intervenção destes profissionais nas estratégias e na rede de atenção à saúde mental, como nos Centros de Atenção Psicossocial (CAPS). No âmbito da formação continuada foram criadas as residências multiprofissionais em saúde mental, com participação de profissionais de Educação Física. Mas, apesar desta inserção resultante das demandas geradas pela própria sociedade, a formação inicial ainda não contempla a adequada discussão do tema e não permite o desenvolvimento de competências que permita aos egressos o entendimento do papel que as atividades físicas podem assumir na atenção à saúde mental. Falta também a criação de oportunidades para a vivência de práticas que possam permitir a preparação para atuação neste novo contexto de intervenção profissional e social. Assim, a proposta de oferecimento desta disciplina procura preencher esta lacuna na formação inicial, avançando na oferta de disciplinas optativas que cumpram o papel de trazer à tona o debate sobre temas emergentes na graduação em Educação Física. Cumpre, dentre outras, a base da flexibilidade na formação, diante da priorização e contemporaneidade na oferta de disciplinas, conforme apontado no Projeto Pedagógico do Curso de Bacharelado.

\section{Ementa}

A saúde mental no Brasil: situação atual e tendências. Inter-relação entre atividade física e saúde mental: evidências clínicas e epidemiológicas. Estratégia de atenção à saúde mental no Brasil e a realidade da intervenção dos profissionais de Educação Física. Os Centros de Atenção Psicossocial como contexto para intervenções da Educação Física. 


\section{Objetivo Geral}

Analisar as possibilidades de intervenção do profissional de Educação Física nos Centros de Atenção Psicossocial e, de modo mais abrangente, na rede de atenção à saúde mental, contextualizando-as em relação à situação de saúde brasileira e ao corpo de conhecimento disponível quanto a inter-relação entre atividade física e saúde mental.

\section{Objetivos específicos}

Espera-se que ao final da disciplina os alunos sejam capazes de: (1) Analisar a situação de saúde mental no Brasil a partir de indicadores básicos disponibilizados pelos Sistemas de Informações em Saúde; (2) Discutir sobre a inter-relação entre atividade física e saúde mental a partir do corpo de conhecimento disponível sobre o assunto; (3) Problematizar as possibilidades de intervenção da Educação Física na área de saúde mental; (4) Identificar possibilidades de atuação do profissional de Educação Física na rede de atenção à saúde mental, em especial nos Centros de Apoio Psicossocial; e, (5) Identificar competências conceituais, atitudinais e procedimentais exigidas do profissional de Educação Física para intervenção na área de saúde mental.

\section{CONTEÚDO}

Durante o desenvolvimento da disciplina, espera-se que sejam tratados os seguintes conteúdos: (1) intervenções da Educação Física na saúde: abordagem histórica e transição de paradigmas; (2) a saúde mental no Brasil: situação atual e tendências; (3) o Sistema Único de Saúde (SUS) e a rede de atenção à saúde mental: história, desenvolvimento e princípios; (4) os Centros de Atenção Psicossocial, seus aspectos organizacionais e normativos; e, (5) a rede de atenção à saúde mental e a intervenção do profissional de Educação Física.

\section{METODOLOGIA}

Para desenvolvimento deste plano de ensino, pretende-se utilizar diferentes procedimentos, incluindo-se a realização de: aulas expositivas dialogadas; atividades de campo (visitas, entrevistas, observação); fichamentos e resenhas de artigos científicos e textos técnicos; produção de textos; e, palestras com convidados externos. Há previsão de que a carga horária de atividades práticas (36 h/a) seja integralmente realizada em vivências e atividades realizadas nos Centros de Atenção Psicossocial da Cidade do Recife. Neste sentido, pretende-se desenvolver estratégia de aprendizagem em serviço mediante colaboração com a Gerência do Programa Academia da Cidade.

\section{AVALIAC̣̃̃O}

A avaliação consistirá de componentes formativos e somativos. Na avaliação formativa será adotada estratégia de autoavaliação, na qual os próprios estudantes serão estimulados a refletir sobre o desempenho alcançado e sobre as competências construídas no decorrer da realização da disciplina. Em relação à avaliação somativa serão utilizados os mesmos procedimentos tanto na primeira quanto na segunda unidade e consistirá dos seguintes elementos: (1) autoavaliação (20\%); (2) teste escrito a ser realizado individualmente e sem consulta, consistindo de questões fechadas que serão elaboradas com base no 
conteúdo programático acumulado até a data de realização da prova (40\%); (3) produção de textos, relatórios e seminários relativos às temáticas discutidas e às atividades práticas realizadas durante a disciplina (40\%).

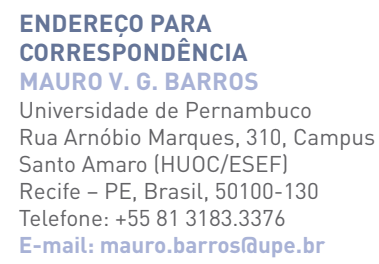

